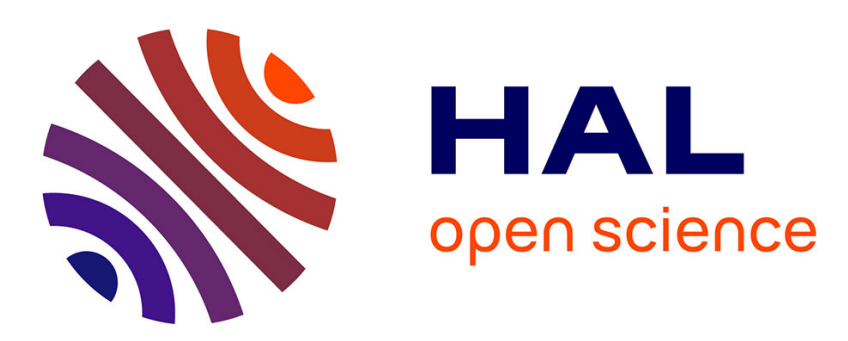

\title{
Synchronization of Traffic Flow and Sector Opening for Collaborative Demand and Capacity Balancing
}

\author{
Yan Xu, Xavier Prats, Daniel Delahaye
}

\section{To cite this version:}

Yan $\mathrm{Xu}$, Xavier Prats, Daniel Delahaye. Synchronization of Traffic Flow and Sector Opening for Collaborative Demand and Capacity Balancing. DASC 2018,37th AIAA/IEEE Digital Avionics Systems Conference, Sep 2018, Londres, United Kingdom. pp.ISBN: 978-1-5386-4113-2, 10.1109/DASC.2018.8569789 . hal-01887290

\section{HAL Id: hal-01887290 \\ https://hal-enac.archives-ouvertes.fr/hal-01887290}

Submitted on 3 Oct 2018

HAL is a multi-disciplinary open access archive for the deposit and dissemination of scientific research documents, whether they are published or not. The documents may come from teaching and research institutions in France or abroad, or from public or private research centers.
L'archive ouverte pluridisciplinaire $\mathbf{H A L}$, est destinée au dépôt et à la diffusion de documents scientifiques de niveau recherche, publiés ou non, émanant des établissements d'enseignement et de recherche français ou étrangers, des laboratoires publics ou privés. 


\section{Synchronization of Traffic Flow and Sector Opening for Collaborative Demand and Capacity Balancing}

\author{
Yan $\mathrm{Xu}$ and Xavier Prats \\ Department of Physics - Aeronautics Division \\ Technical University of Catalonia \\ Castelldefels 08860, Barcelona, Spain
}

\author{
Daniel Delahaye \\ OPTIM Group, ENAC Laboratory \\ École Nationale de l'Aviation Civile \\ 7 Av. Edouard Belin, 31400 Toulouse, France
}

\begin{abstract}
This paper proposes a method to synchronize traffic flow optimization and sector opening scheduling, with the aim of achieving flexible demand and capacity balancing (DCB). Delay assignment, trajectory options and sector collapsing are used to manage the traffic demand, while sector opening schemes are to affect the airspace capacity. Mixed Integer Programming (MIP) model is built to incorporate these initiatives. Three model variants are presented to illustrate the synchronization process, and their results in a real-world case study demonstrate some promising improvements for the DCB performances.
\end{abstract}

Index Terms - air traffic flow management, trajectory options, dynamic sectorization, demand and capacity balancing

\section{NOMENCLATURE}

\begin{tabular}{ll}
$f \in F$ & set of flights \\
$j \in J$ & set of elementary sectors \\
$k \in K$ & set of trajectory options \\
$t \in T$ & set of time moments \\
$\tau \in \mathcal{T}$ & set of time periods \\
$l \in L$ & set of operating sectors \\
$a \in A$ & set of area control centers \\
$s \in S$ & set of airspace configurations \\
$K_{f}$ & subset of trajectory options submitted by $f$ \\
$J_{f}^{k}$ & subset of elementary sectors that $f$ (or $k$ ) traverses \\
$T_{f}^{k, j}$ & subset of time feasible for $f$ (or $k$ ) entering $j$ \\
$L_{\tau}$ & subset of operating sectors that are open in $\tau$ \\
$L_{j}$ & subset of operating sectors constructed by $j$ \\
$J_{l}$ & subset of elementary sectors consisted in $l$ \\
$S_{l}$ & subset of airspace configurations associated with $l$ \\
$S_{a}$ & subset of airspace configurations belonged to $a$ \\
$J_{f, l}^{k, \tau}$ & first elementary sector that $f$ (or $k$ ) traverses and \\
$\bar{T}_{f}^{k, j}$ & that operates in $l$ in $\tau$ \\
$T_{f}^{k, j}$ & upper bound of feasible time window $T_{f}^{k, j}$ \\
$r_{f}^{k, j}$ & lower bound of feasible time window $T_{f}^{k, j}$ \\
$n_{f}^{k}$ & estimated arrival time of $f$ (or $k$ ) entering $j$ \\
$\hat{t}_{f}^{k, j j^{\prime}}$ & number of elementary sectors that $f$ (or $k$ ) traverses \\
$c_{l}^{\tau}$ & scheduled flight time of segment $j j^{\prime}$ for $f$ (or $k$ ) \\
$d_{f}^{k}$ & operating capacity of $l$ in $\tau$ \\
$e_{f}^{k}$ & extra fuel consumption for $k$ of $f$ \\
$\alpha$ & extra route charges for $k$ of $f$ \\
$\gamma$ & unit cost of ground delay \\
$\delta$ & unit cost of fuel consumption \\
$M$ & unit cost of opening an operating sector \\
\hline & artificial parameter of large positive value \\
\hline &
\end{tabular}

This paper was partially funded by grants from the CSC No 201506830050 and by the SESAR Joint Undertaking under grant agreement No 699338 .

\section{INTRODUCTION}

The air transportation system currently faces a significant strain from the fast-growing flight demand. This has been evidenced in recent years by severe flight delays and more commonly-seen network congestions. In Europe, year 2016 saw an average departure delay per flight of 11.3 minutes (and 29.1 minutes, per delayed flight, for the average arrival delay), an increase of $9 \%$ in comparison to 2015. Furthermore, flights delayed more than 30 minutes from all-causes increased to $9.8 \%$, while a monthly average of $1.9 \%$ of operational cancellation occurred [1].

One of the primary causes for those delays and congestions is that the number of flights (demand) often exceeds the supply of the airspace accommodation (capacity). Convective weather, airspace restrictions, overloaded airports/sectors and air traffic control (ATC) industrial actions can temporarily reduce the supply, in addition to the sustained increase of air traffic volume during a relatively long-term period, imposing, from both sides, the imbalances between demand and capacity. The effort thereby to balance demand with capacity is typically known as air traffic flow management (ATFM) [2].

Following the pioneering work done in [3], a number of researchers have focused their activity on the development of optimization models for the delay assignment as a short-term measure for ATFM regulations (see [4] for instance). Further taking into account the capacity constraints from airspace sectors, the problem of controlling release times and speed adjustments of aircraft while airborne for a network of airports (including sectors) has been studied in [5], [6].

Examples of sophisticated ATFM initiatives include Ground Delay Programs (GDPs) and Airspace Flow Programs (AFPs) in the United States [7]. Similar initiatives exist in Europe, implemented by the Eurocontrol's Network Manager Operations Centre (NMOC, previously called CFMU). On basis of the existing GDP/AFP, a newly-introduced Collaborative Trajectory Options Program (CTOP) has been deployed in the U.S. since 2014, which could tackle multiple flow constrained areas within a single program and allow airlines to submit a set of preferred trajectory options, i.e., Trajectory Options Set (TOS), in prior to the issuance of the program [8].

On the other hand, the airspace system nowadays is typically partitioned into sectors, each of which is handled by a group 
of air traffic controllers and is bonded with a limited capacity. However, as pointed out in [9], some of these capacity resources might be under-utilized and lack of flexibility, and thus requires a better reorganization. Relevant studies encompass dynamic airspace configuration (DAC), in which airspace (capacity) is adjusted in real time to accommodate the demand that may change throughout a given day [10]. In addition, several challenges with respect to the DAC was noted in [11], and a more flexible approach was proposed that designs the shareable sub-sections of the current airspace configuration.

In this paper, we present three model variants to demonstrate the synchronization of traffic flow optimization and sector opening scheduling. The first model variant is focused on regulating the traffic demand by means of assigning delays. Then, alternative trajectory options, which is similar to the concept of TOS, is further added to the second model variant, in such a way that the traffic flow patterns can be managed in both time and space domains. Next, the third model variant relaxes the constraints of airspace structures (and capacities) that are fixed in the previous two models, allowing (limited) flexible sector opening. All the three models are aimed at achieving demand and capacity balancing (DCB), but their performances differ significantly. Through comparing their results in a realistic case study, we show the notable benefits of using the proposed synchronization method.

\section{Motivation}

In previous work [12], we discussed an approach of combining different delay management initiatives into an integrated optimization model, in such a way that the performance of demand and capacity balancing (DCB) can be improved. Based on this work, we demonstrated in [13] a large delay reduction with the DCB algorithm when further incorporating airlines' alternative trajectory options. However, all these measures are

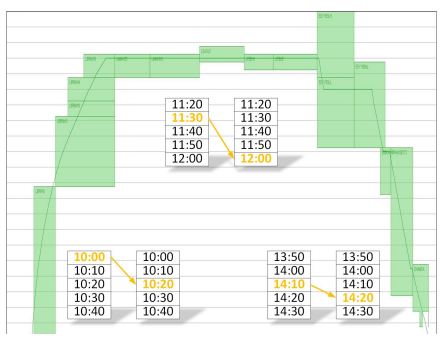

(a) Assigning delays

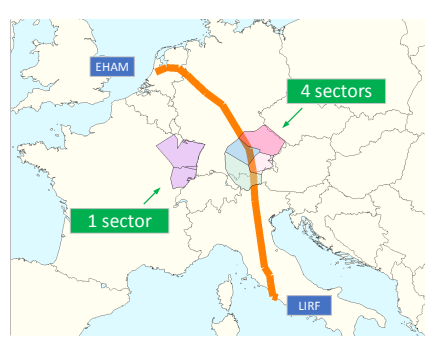

(c) Fixed airspace structures

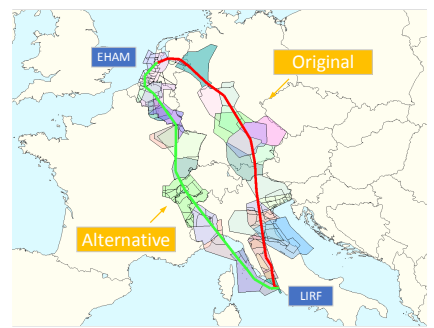

(b) Allowing alternative trajectories

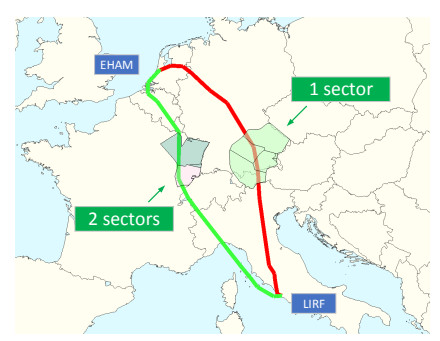

(d) Adjusting airspace structures
Fig. 1. Intuition of measures for balancing demand and capacity. aimed at managing the traffic demand by means of regulating the traffic flow, namely assigning delays in different ways (see Fig. 1(a)) and allowing alternative trajectories (see Fig. 1(b)). The airspace structures, at the same time, are always fixed.

Subsequently, one implicit problem is that the given airspace structures (and corresponding capacities) are often designed to best accommodate the traffic flow patterns that are accumulated by the planned (or historical) flight trajectories. In other words, once these trajectories have been changed, either by imposing delays [12] or diverting to the alternatives [13], the temporal-spatial flow patterns will change too, and thus the initial airspace structures may turn to be not optimal.

Let us take a look at an example in Fig. 1(c) and Fig. 1(d) where the airspace structure is fixed and is subject to flexible adjustment respectively. For the original flight plans, more airline operators prefer to schedule a flight route, as colored in red, to fly from Rome (LIRF) to Amsterdam (EHAM). Accordingly, for the two areas labeled out in the map, as shown in Fig. 1(c), the one crossed by the congested route is divided to 4 sectors (for instance) operating at the same time to provide more capacities, whilst the other area is run by only 1 sector as a whole. However, having been through the abovementioned DCB algorithm, some flights could be diverted to the route colored in green (see Fig. 1(d)), and some flights could be assigned with certain delays such that their Controlled Times of Arrival (CTAs) at that area would be changed (and sequenced) as well. That is to say, the previous congested area could become less demanded of capacities. Therefore, it would be beneficial to merge the former 4 sectors to 1 entire sector to reduce the extra ATC costs, and, meanwhile, to diverge the previously less-congested area from 1 sector to 2 smaller sectors to better handle the additional traffic.

Following this thought, we will present in this paper 3 mixed integer programming (MIP) model variants to illustrate the effects of synchronizing demand (i.e., traffic flow) and capacity (i.e., sector opening) for the DCB algorithm. They are respectively:

- Model DCB: assigning delays;

- Model C-DCB: assigning delays, and allowing alternative trajectory options; and

- Model SC-DCB: assigning delays, allowing alternative trajectory options, and adjusting sector opening schemes.

The first model uses only delay assignment, which serves as the baseline of this paper. The second model further includes alternative trajectories, and provides key benchmark results. Based on them, the third model considers the synchronization issue and gives some promising findings.

\section{MODEL DCB}

Model DCB aims at balancing traffic demand under capacity through assigning ground delays to certain flights. The demand is counted according to the 4-Dimensional (4D) trajectories initially scheduled by the airlines. Meantime, the capacity is considered in form of aircraft entry rate. It is determined by the fixed airspace structures and the airspace entities' unit capacity 
values, which, in turn, were planned and evaluated well in advance based on the historical data.

\section{A. Decision variables}

Aiming at future trajectory based operations (TBO), delays are imposed in this paper at each control point along the trajectory, with the CTA concept, which is defined at each entrance position of airspace entity (i.e., elementary sector) that the trajectory is scheduled to traverse. Subsequently, in order to assign the CTAs, we consider a set of decision variables as follows:

$$
x_{f, t}^{j}= \begin{cases}1, & \text { if flight } f \text { arrives at elementary sector } j \text { 's } \\ \text { entrance by time } t \\ 0, \quad \text { otherwise }\end{cases}
$$

It should be noted that the "by" time is used, rather than "at" as the decision variables, which would enable a faster solution searching time as introduced in [2], while the "at" time can be derived by $\left(x_{f, t}^{j}-x_{f, t-1}^{j}\right)$. Other than the model presented in [12], where various cost-based delay measures were adopted, we consider only ground holding in this paper. The reason is because ground holding is still the cheapest and commonlyused way to absorb delays. Besides, the inclusion of other measures will not essentially change the DCB synchronization process, and thus is out of the scope of this paper.

\section{B. Model formulation}

$$
\begin{aligned}
& \min \sum_{f \in F} \sum_{j=J_{f}(1)} \sum_{t \in T_{f}^{j}}\left(t-r_{f}^{j}\right)\left(x_{f, t}^{j}-x_{f, t-1}^{j}\right) \\
& \text { s.t. } \quad x_{f, \underline{T}_{f}^{j}-1}^{j}=0, x_{f, \bar{T}_{f}^{j}}^{j}=1 \quad \forall f \in F, \forall j \in J_{f} \\
& x_{f, t}^{j}-x_{f, t-1}^{j} \geq 0 \quad \forall f \in F, \forall j \in J_{f}, \forall t \in T_{f}^{j} \\
& x_{f, t+\hat{t}_{f}^{j j^{\prime}}}^{j^{\prime}}-x_{f, t}^{j}=0 \quad \forall f \in F, \forall t \in T_{f}^{j}, \\
& j=J_{f}(i), j^{\prime}=J_{f}(i+1): \forall i \in\left[1, n_{f}\right) \\
& \sum_{f \in F} \sum_{j=J_{f, l}^{\tau}} \sum_{t \in T_{f}^{j} \cap \tau} x_{f, t}^{j}-x_{f, t-1}^{j} \leq c_{l}^{\tau} \\
& \forall l \in L_{\tau}, \forall \tau \in \mathcal{T} \\
& x_{f, t}^{j} \in 0,1 \quad \forall f \in F, \forall j \in J_{f}, \forall t \in T_{f}^{j}
\end{aligned}
$$

The objective function (1) of Model DCB is simply to minimize the total amount of delay assignments. Fairness issues could be partially taken into account by adding a small super-linear factor $\epsilon(\epsilon>0)$ to the cost of delay for each flight, namely $\left(t-r_{f}^{j}\right) \rightarrow\left(t-r_{f}^{j}\right)^{1+\epsilon}$. In this way, delays will be assigned moderately across all the flights, instead of unevenly to one particular flight. However, as the equilibrium criteria is relatively subjective and also proves to have notable trade-offs with the system efficiency [14], it deserves another separate discussion. For convenience, we set $\epsilon$ to 0 for all the 3 model variants in this paper, focusing only on the overall costs.

Constraint (2) specifies the boundary of CTA at each control point for each flight, i.e., $T_{f}^{j}$, which depends on the corresponding Estimated Time of Arrival (ETA) added with an allowable maximal amount of delay. Constraint (3) guarantees the timeline continuity of the decision variables. Since we allow ground delay only, constraint (4) ensures that the airborne (segment) flight time for the controlled flight assigned with CTAs will still remain the same as the initially scheduled. Then, the airspace capacity constraints are enforced in (5), where we may notice an inconsistence between the capacity entity (i.e., operating sector $l$ ) and the control point (i.e., elementary sector $j$ ). To solve this issue, we follow the commonly-used rule that, for each flight, only the first entry (control point) into an operating sector is counted, namely $j=J_{f, l}^{\tau}$. The remaining entries (if any) inside this operating sector during the same period will be regarded as internal activities, not another traffic demand. Finally, all decision variables are subject to Constraint (6).

\section{MODEl C-DCB}

Model Collaborative DCB (C-DCB) is based on the previous Model DCB but requires more contributions from the airlines. Besides the submission of the initially planned trajectories, airlines are allowed in this model to submit a number of alternative trajectories for their affected flights in order to route out of the detected hotspot areas. Model C-DCB will then decide which is the best distribution of trajectory selections and delay assignments for all the flights based on a centralized global optimization.

\section{A. Hotspot avoidance}

Given the planned flight trajectories and airspace structures (and corresponding entity capacities), we can assess the initial demand and capacity balancing situations, and subsequently identify the hotspot areas (where demand is greater than capacity) as well as the associated flights. As both flight

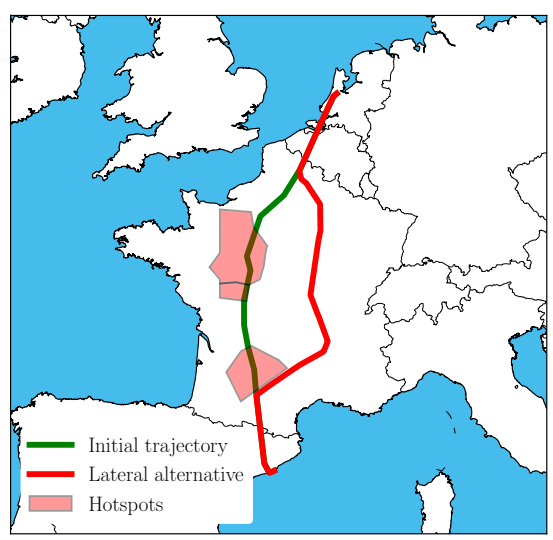

Fig. 2. Initial trajectory (green line) traversing two identified hotspots (red polygons) and an alternative trajectory (red line) laterally bypassing them with fewest extra costs. 


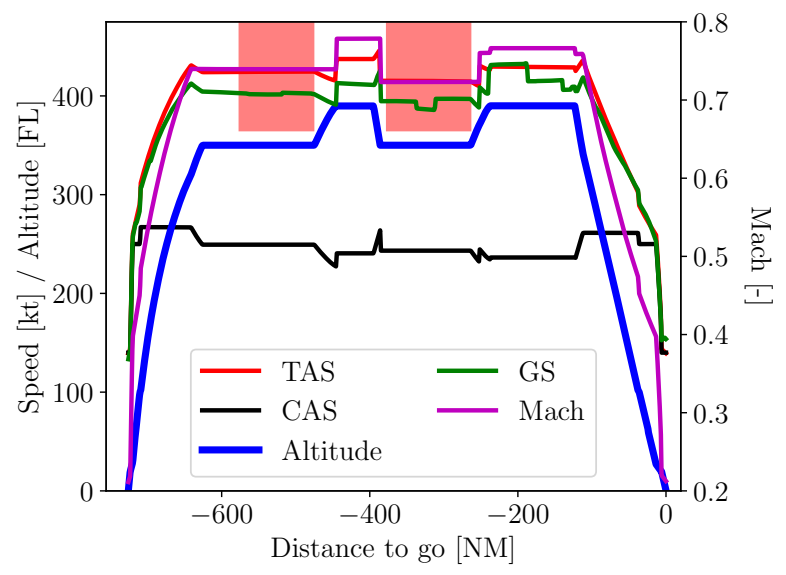

Fig. 3. An alternative trajectory (blue line) vertically avoiding the two identified hotspots (red polygons) with fewest extra costs.

trajectory and airspace structure are time-dependent, it is clear that the hotspot areas will be also time-varying. Although there might be several hotspot sectors detected in the network for the same time period, the captured flights only need to bypass the sectors that they are scheduled to traverse (see Fig. 2), without taking into account the other hotspots.

However, rerouting could be too expensive to be adopted by airline operators, such that their incentives of participation in this Collaborative DCB decision-making process may be reduced. Thus, with the objective of incurring as few extra costs as possible (compared with the initial trajectory), we provide additional hotspot-avoidance information to the airlines for each of their captured flights. Furthermore, as airspace sectors are typically defined in the 3-Dimensional space, it is not only changing the flight path laterally that works but also adjusting vertically the flight altitude (as well as their combinations). Consequently, the avoidance information will contain both the lateral and vertical cases.

For the lateral avoidance, as shown in Fig. 2, the boundary coordinates of each airblock are given in such a way that a specific polygon graph can be formed on the horizontal plane to represent each hotspot area. The alternative trajectory must avoid to intersect with any of the connecting frontiers. For the vertical-avoidance, as shown in Fig. 3, we inform the flight at which distance (to the destination airport, e.g., -590 $\mathrm{nm}$ ) it should start to change the initial altitude and at which distance (e.g., $-480 \mathrm{~nm}$ ) to recover that altitude (if desired), as well as the non-selectable flight levels (e.g., from FL365 to FL999) between the two distances, for each sector that the initial trajectory needs to avoid.

\section{B. Trajectory options}

The key difference of Model C-DCB compared to Model DCB is that it allows more trajectory options for every single flight, rather than only having the initially planned. In the previous Sec. IV-A, we have introduced a way of sharing the hotspot-avoidance information, aiming to assist airlines to
TABLE I

POSSIBLE TRAJECTORY OPTIONS SUBMITTED FOR ONE FLIGHT.

\begin{tabular}{c|c|c}
\hline Trajectory options & Extra costs & Comments \\
\hline Trajectory 0 & 0 & Initial trajectory \\
\hline Trajectory 1 & Cost 1 & Lateral hotspot avoidance \\
\hline Trajectory 2 & Cost 2 & Vertical hotspot avoidance \\
\hline Trajectory 3 & Cost 3 & Updated wind field prediction \\
\hline Trajectory 4 & Cost 4 & Experienced congestion areas \\
\hline$:$ & $\vdots$ & $\vdots$ \\
\hline Trajectory $\mathrm{n}$ & Cost $\mathrm{n}$ & Any specific preferences
\end{tabular}

schedule their alternative trajectories with as few extra costs as possible. Nevertheless, it must be noted that the submission of any alternative is not mandatory, and should be subject to the airlines' independent decisions. The operators could always keep the original one only, if for example the extra costs of all the feasible alternatives are relatively high.

On the other hand, if the extra costs are quite small, the flight simply needs to change its cruise flight level slightly such that two hotspot sectors can be avoided (see Fig. 3), then it might be worthwhile to submit it because in this way its potential delays might be avoided. In addition, if there exist some other flight who can bypass the two hotspots with even less extra costs required, then the model would more often decide to divert that flight, which means that this flight could probably still use its initial trajectory even though it has submitted the alternatives.

Besides, not only the above hotspot-avoidance trajectories, but also some others of specific purposes can be submitted as alternatives as well. For example, as time elapsed the weather forecast usually turns more accurate, and therefore it would be appropriate to schedule a new trajectory taking advantage of the latest predictions such as the wind field condition.

To sum up, no matter for what reasons, airlines are allowed to freely submit a set of preferred trajectories in this model (see Table I), and are also required to label out the corresponding costs with respect to their initial trajectories. However, how to model these costs in an effective way is still under our assessment, as in most cases the airlines' cost information is still proprietary, and misusing of this information may also lead to vicious competition issues in realistic operations.

\section{Decision variables}

Similar to the time-related decision variables $x_{f, t}^{j}$ defined previously in Model DCB, we include an extra domain $k$ representing the trajectory options in this model, as follows:

$$
x_{f, t}^{k, j}= \begin{cases}1, & \text { if flight } f \text { 's } k \text { th trajectory arrives at } \\ \text { elementary sector } j \text { 's entrance by time } t \\ 0, & \text { otherwise }\end{cases}
$$

In this case, all the control points and associated CTAs are bonded to the $k$ th trajectory, instead of the flight itself. Therefore, in order to connect between them, we consider an additional set of decision variables $z_{f}^{k}$ to tell if the $k$ th 
trajectory is eventually selected for that flight $f$, namely:

$$
z_{f}^{k}= \begin{cases}1, & \text { if flight } f \text { 's } k \text { th trajectory is selected } \\ 0, & \text { otherwise }\end{cases}
$$

The two sets of variables are linked together by a following constraint, in such a way that delays are still imposed on each particular flight, rather than any of its unselected trajectories.

\section{Model formulation}

$$
\begin{aligned}
& \min \sum_{f \in F} \sum_{k \in K_{f}} \sum_{j=J_{f}^{k}(1)} \sum_{t \in T_{f}^{k, j}} \alpha\left(t-r_{f}^{k, j}\right)\left(x_{f, t}^{k, j}-x_{f, t-1}^{k, j}\right) \\
& +\sum_{f \in F} \sum_{k \in K_{f}}\left(\gamma d_{f}^{k}+e_{f}^{k}\right) z_{f}^{k} \\
& \text { s.t. } \quad \sum_{k \in K_{f}} z_{f}^{k}=1 \quad \forall f \in F \\
& x_{f, \underline{T}_{f}^{k, j}-1}^{k, j}=0, x_{f, \bar{T}_{f}^{k, j}}^{k, j}=z_{f}^{k} \quad \forall f \in F, \\
& \forall k \in K_{f}, \forall j \in J_{f}^{k} \\
& x_{f, t}^{k, j}-x_{f, t-1}^{k, j} \geq 0 \quad \forall f \in F, \forall k \in K_{f}, \\
& \forall j \in J_{f}^{k}, \forall t \in T_{f}^{k, j} \\
& x_{f, t+\hat{t}_{f}^{k, j j^{\prime}}}^{k, j^{\prime}}-x_{f, t}^{k, j}=0 \quad \forall f \in F, \forall k \in K_{f}, \forall t \in T_{f}^{k, j}, \\
& j=J_{f}^{k}(i), j^{\prime}=J_{f}^{k}(i+1): \forall i \in\left[1, n_{f}^{k}\right) \\
& \sum_{f \in F} \sum_{k \in K_{f}} \sum_{j=J_{f, l}^{k, \tau}} \sum_{t \in T_{f}^{k, j} \cap \tau} x_{f, t}^{k, j}-x_{f, t-1}^{k, j} \leq c_{l}^{\tau} \\
& \forall l \in L_{\tau}, \forall \tau \in \mathcal{T} \\
& x_{f, t}^{k, j} \in 0,1 \quad \forall f \in F, \forall k \in K_{f}, \forall j \in J_{f}^{k}, \forall t \in T_{f}^{k, j} \\
& z_{f}^{k} \in 0,1 \quad \forall f \in F, \forall k \in K_{f}
\end{aligned}
$$

The objective function (7) of Model C-DCB is to minimize the total delay costs and the extra costs incurred from altering trajectories. We consider the fuel consumption $\left(d_{f}^{k}\right)$ and route charges $\left(e_{f}^{k}\right)$ in this paper as the main trajectory-related costs, but in realities more detailed costs could be further taken account and thus specified by airlines.

Constraint (8) ensures that only one trajectory is selected for each flight from the set of its submitted trajectory options $\left(K_{f}\right)$. Constraint (9) and (10) seem similar to those in Model $\mathrm{DCB}$, but the difference is that the value at the upper bound of feasible time window $\left(\bar{T}_{f}^{k, j}\right)$ is dependent on trajectory selection $\left(z_{f}^{k}\right)$. The two constraints further enforce that if a trajectory is not selected, i.e., $z_{f}^{k}=0$, then all its associated time variables are equal to 0 , meaning that no CTA would be assigned to any of the control points along that trajectory.
Constraints (11) and (12) remain the same functions as (4) and (5) have, which respectively guarantees the segment flight time and stipulates demand not to exceed the planned capacity provisions. Finally, Constraints (13) and (14) specify the set domains and state that all decision variables are binary.

\section{MODEL SC-DCB}

Model Synchronized Collaborative DCB (SC-DCB) is to relax the hard constraint of airspace structures fixed in both Model DCB and Model C-DCB. As mentioned before, delays and alternative trajectories are used to regulate the 4-D traffic flow, which may lead to severe unfitness to the initially planned airspace structures. This model maintains all previous traffic management initiatives but also adjusts (if needed) the sector opening schemes, trying to balance the traffic demand and airspace capacity in a synchronized way.

\section{A. Airspace structure and capacity}

According to the European airspace structure, as shown in Fig. 4, a large piece of airspace typically consists of several Area Control Centers (ACCs). Under current operations, each ACC normally runs independently, and has its own limited amount of configurations and the corresponding opening schemes. Next, each specified configuration is composed of several elementary sectors and/or collapsed sectors, and each collapsed sector is in turn merged by several smaller elementary sectors. In addition, the small elementary sector is further defined by a certain number of basic airblock volumes, as well as the specific bottom and top flight levels.

However, given the basic airblock volumes typically remain stable in a relatively long period (and this paper is focused on the pre-tactical phase), we consider elementary sectors being the smallest entities in this model. Consequently, we can imagine an entire 3-D block of airspace filled by a network of non-overlapping elementary sectors (see Fig. 5). With the timeline added in a 4-D scenario, sometimes a small elementary sector itself functions as an operating sector, and at

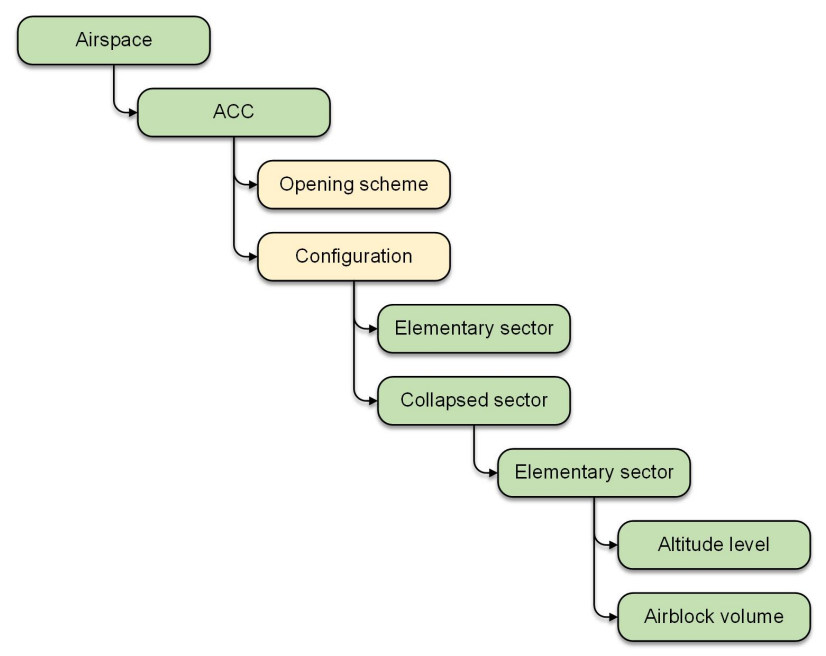

Fig. 4. Schematic of airspace structure implemented in the Eurocontrol area. 


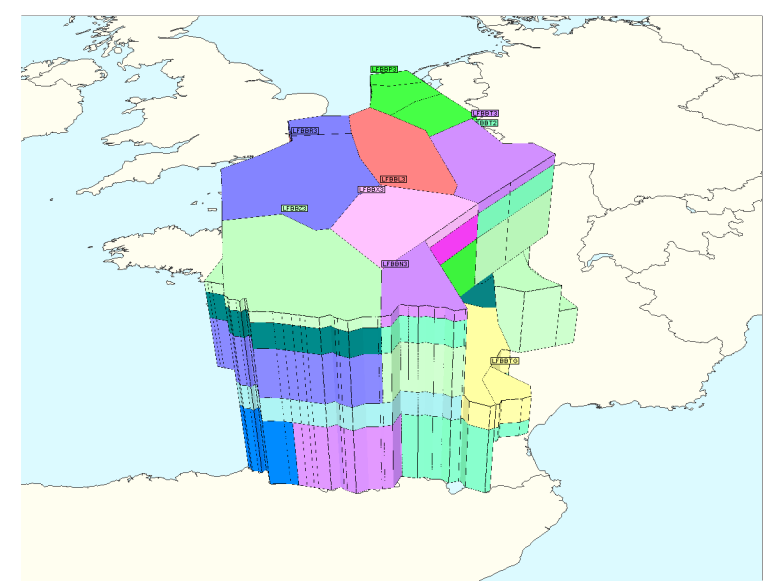

Fig. 5. Airspace filled with non-overlapping elementary sectors, some of which can be merged and operated as a whole during certain time periods depending on the traffic situation.

another time it is merged into a larger collapsed sector that acts as another operating sector. Moreover, each operating sector is bonded with a certain operating capacity, which in turn could be associated with several Traffic Volumes. Yet, only one of them should be activated at a time. For more details about retrieving this information, the reader may direct to [12].

\section{B. Flexible sector opening}

Let us recall the overall airspace structure shown in Fig. 4. There could be several ways to realize dynamic airspace reconfiguration (on different levels). For example, change the opening scheme of each elementary sector, and allow any adjacent elementary sectors to collapse, which then act as a whole as an operating sector (see Fig. 5). In this case, some unknown collapsed sectors might be created. More concretely, it is also possible to modify the physical dimension of the

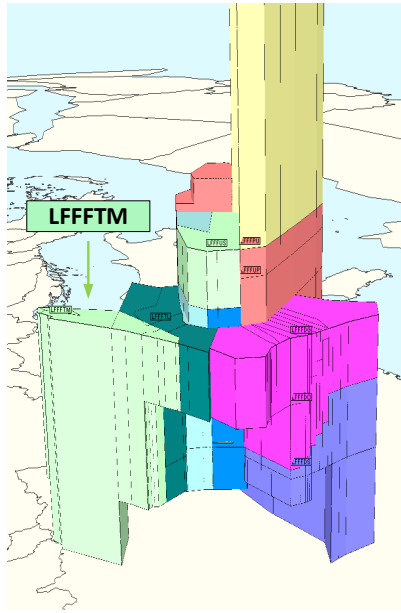

(a) LFFFCTAE: Conf. 10B

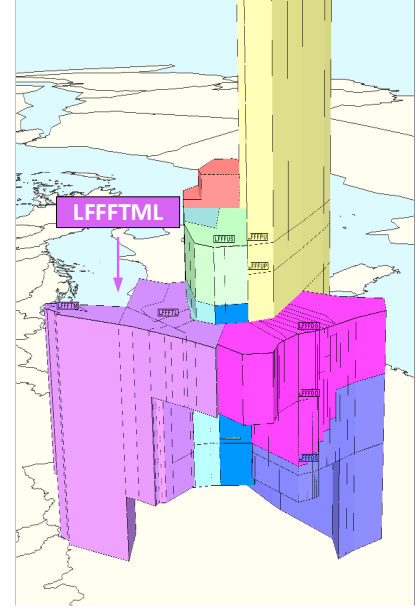

(b) LFFFCTAE: Conf. 10F
Fig. 6. Operating sectors consisted in two different configurations $10 \mathrm{~B}$ and $10 \mathrm{~F}$ for ACC LFFFCTAE. elementary sector (along with its opening scheme). Namely, design new elementary sectors based on the basic airblock volumes. Furthermore, even the smallest airblock volumes could be reshaped as well, according to the traffic flow pattern or complexity appearing in that specific area for instance.

In general, the above methods could provide a precise insight into the dynamic sectorization problem, and thus result in efficient airspace reconfiguration. On the other hand, however, incorporating such complicated approaches, i.e., creating unknown collapsed/elementary sectors or airblocks, into the original model presented in this paper may largely increase its computational burden. Note that the model is aimed to optimize not only the sectorization but also trajectory selection and delay assignment in the meantime.

Therefore, in this paper we explore a less ambitious dynamic sectorization method, scheduling the opening schemes for the already existing operating sectors, without changing the shape of any collapsed and/or elementary sectors. To further reduce the computational complexity, we specify that the opening schemes of sectors are subject to the existing airspace configurations. We can see an example in Fig. 6, where elementary sector LFFFTM acts as an independent operating sector (light green block in Fig. 6(a)) in configuration "10B" of ACC LFFFCTAE, while it is merged into a collapsed sector LFFFTML (purple block in Fig. 6(b)) when configuration "10F" is activated.

The effects of this limited dynamic sectorization are three folded: 1) it enables flexible capacity provision, which means that capacities can be re-allocated from free areas to the newlyemerging congested areas (caused by traffic flow regulation); 2) it allows the adjustment of collapsing architecture of the elementary sectors, which can affect the traffic demand counting for operating sectors (recall Constraint (5) and relevant statements); and 3) it takes the number of opening sectors into account, which is related with ATC system costs.

\section{Decision variables}

Following the above discussion, we define an additional set of decision variables as follows, along with $\left(x_{f, t}^{k, j}\right)$ and $\left(z_{f}^{k}\right)$ that have been already introduced in Model C-DCB.

$$
u_{s}^{\tau}= \begin{cases}1, & \text { if configuration } s \text { is activated in time period } \tau \\ 0, & \text { otherwise }\end{cases}
$$

Note that once an airspace configuration $(s)$ is settled, the status of its associated operating sectors $(l)$ should be also determined. In other words, the following set of variables $\left(w_{l}^{\tau}\right)$ representing each individual sector's opening:

$$
w_{l}^{\tau}= \begin{cases}1, & \text { if sector } l \text { is open during time period } \tau \\ 0, & \text { otherwise }\end{cases}
$$

can be replaced by $\left(w_{l}^{\tau}=\sum_{s \in S_{l}} u_{s}^{\tau}\right)$ for all sectors and time periods, where $\left(S_{l}\right)$ is the configurations constructed (partially) by sector $(l)$. In this case, if any of the airspace configurations related with sector $(l)$ is activated (i.e., $\sum_{s \in S_{l}} u_{s}^{\tau}=1$ ), then this sector must be open; On the contrary, if the sector is not 
open (i.e., $w_{l}^{\tau}=0$ ), then it means that all the configurations $\left(S_{l}\right)$ constructed by this sector cannot be activated.

For convenience, the time period $(\tau)$ is defined with the same length as the unit time scale used for demand counting and capacity provision. A smaller scale may also apply, but requires an equivalent capacity value to match with it.

\section{Model formulation}

$$
\begin{gathered}
\min \sum_{f \in F} \sum_{k \in K_{f}} \sum_{j=J_{f}^{k}(1)} \sum_{t \in T_{f}^{k, j}} \alpha\left(t-r_{f}^{k, j}\right)\left(x_{f, t}^{k, j}-x_{f, t-1}^{k, j}\right) \\
+\sum_{f \in F} \sum_{k \in K_{f}}\left(\gamma d_{f}^{k}+e_{f}^{k}\right) z_{f}^{k}+\sum_{l \in L} \sum_{s \in S_{l}} \sum_{\tau \in \mathcal{T}} \delta u_{s}^{\tau} \\
\text { s.t. } \quad(8)-(11) \text { and (13)-(14) } \\
\sum_{s \in S_{a}} u_{s}^{\tau}=1 \quad \forall a \in A, \forall \tau \in \mathcal{T} \\
\sum_{s \in S_{l}} u_{s}^{\tau} \leq 1 \quad \forall l \in L, \forall \tau \in \mathcal{T} \\
\sum_{l \in L_{j}} \sum_{s \in S_{l}} u_{s}^{\tau}=1 \quad \forall j \in J, \forall \tau \in \mathcal{T} \\
\sum_{f \in F} \sum_{k \in K_{f}} \sum_{j=J_{f, l}^{k, \tau}} x_{f \in T_{f}^{k, j} \cap \tau}^{k, j}-x_{f, t-1}^{k, j} \leq \sum_{s \in S_{l}} c_{l}^{\tau} u_{s}^{\tau} \\
+\left(1-\sum_{s \in S_{l}} u_{s}^{\tau}\right) M \quad \forall l \in L, \forall \tau \in \mathcal{T} \\
u_{s}^{\tau} \in 0,1 \quad \forall s \in S, \forall \tau \in \mathcal{T}
\end{gathered}
$$

The multi-objective function (15) minimizes three groups of costs, including the costs of total delays, the extra costs of using alternative trajectories (e.g., fuel consumption and route charges), and the ATC operating costs which are dependent on the total number of opened sectors.

Since Model SC-DCB maintains all the traffic management initiatives used in Model C-DCB, the corresponding Constraints (8)-(11) and (13)-(14) are also required in this model. Besides, Constraint (16) guarantees that for each ACC there must be one configuration (among all the selectable configurations $\left(S_{a}\right)$ associated with the specific ACC) activated in each time period. Constraint (17) ensures that each operating sector can function in only one particular configuration for the maximal during one time period. Next, Constraint (18) stipulates that all the elementary sectors in airspace should be "in use" (i.e., providing services) no matter how they are collapsed in different ways (recall Fig. 5).

In Constraint (19) concerning the capacities, the left-hand term looks just the same as that appearing in Constraint (12). For the right-hand term, since we have no idea which sector will be open before executing the model, we should consider the capacity for all the possible operating sectors $(L)$, rather than a subset $\left(L_{\tau}\right)$ that are known and fixed in previous models. However, if one specific sector is not open, we will not expect it to become the reason of assigning delays for instance. Therefore, a large positive value $(M)$ is added to the right-hand term. By doing this, if it is not open (i.e., $1-w_{l}^{\tau}=1$ ), the inequality of Constraint (19) is still satisfied and there is no need to regulate the traffic flow. Finally, Constraints (20) states that the additional set of decision variables are binary.

\section{COMPUTATIONAL EXPERIMENTS}

Computational experiments have been performed with the three model variants presented in this paper. Real-world data are used for the experiments. Results are compared with respect to the three variants, which illustrates the proposed synchronization process and show its significant effects on improving the DCB performances.

\section{A. Experimental setup}

The experiment is focused on the French airspace with 24 hours' traffic traversing this area. Specifically, it includes 6,255 planned flights, 15 ACCs, 1,511 configurations, 164 elementary sectors and the associated 431 existing operating sectors. In this study, we consider $1 \mathrm{~min}$ as the unit time step, and $60 \mathrm{~min}$ as the time scale for demand counting. All this information is retrieved from the Demand Data Repository version 2 (DDR2) published by Eurocontrol for a typical day in February in 2017. The flight trajectories, on the other hand, are generated according to their flight plans by an in-house trajectory planning tool [15].

The above presents the generic setup for all case studies in this paper. However, for Model DCB, since the sector opening schemes are known (according to the DDR2 database) and fixed, the number of possible operating sectors decreases to 224 in total across the day. Next, for Model C-DCB, the airspace settings are the same as those for Model DCB. In addition, with 86 time-varying hotspot areas identified (see Sec. IV-A), there are 1,305 lateral and 1,379 vertical alternative trajectories further scheduled and submitted (see Sec. IV-B). Therefore, we have 8,939 trajectories in total for the planned 6,255 flights. Finally, for Model SC-DCB, it remains the generic airspace setup and also takes all the submitted trajectories from Model C-DCB into account.

Some key assumptions and parameters have been taken: 1) the unit cost of delay $(\alpha)$ keeps constant (i.e., 5 euro/min arbitrary value) and applies the same for different flights; 2 ) the unit cost of fuel consumption $(\gamma)$ is 0.5 euro $/ \mathrm{kg} ; 3)$ the route charges are calculated according to the absolute distance

TABLE II

PROBLEM SIZE AND COMPUTATIONAL TIME.

\begin{tabular}{c|c|c|c}
\hline Summary & Model DCB & Model C-DCB & Model SC-DCB \\
\hline Time win. (min) & 360 & 120 & 20 \\
\hline Variables & $12,392,347$ & $6,413,940$ & $1,520,268$ \\
\hline Equations & $22,459,267$ & $11,685,916$ & $2,413,425$ \\
\hline Non-zeros & $47,518,810$ & $24,718,752$ & $6,169,484$ \\
\hline Generation (min) & 2 & 1 & 1 \\
\hline Solution (min) & 4 & 2 & 51 \\
\hline
\end{tabular}


flown; 4) airlines are willing to share the detailed costs of their alternative trajectories; 5 ) the unit cost of opening a sector $(\delta)$ for $60 \mathrm{~min}$ is 100 euro; 6) the time scale $(\tau)$ for demand counting and capacity is $60 \mathrm{~min}$; and 7) the maximal allowance of capacity overload is set to $10 \%$.

In addition, since the amount of delays required are quite different, we set different feasible time windows $T_{f}^{k, j}$ in the three models, which in turn affects notably the problem dimensions, as listed in Table II. In the numerical experiments, GAMS v.25.0 software suite has been used as the modeling tool and Gurobi v.7.5 optimizer has been used as the solver. The numerical experiments have been run on a 64 bit Intel i7-4790@3.60 GHz quad core CPU computer with 16 GB of RAM memory and Linux OS.

The model generation time and solution time are presented in Table II as well. The integrity relative gap is set to $0 \%$. We can notice that even though Model SC-DCB is much smaller than the other two models (because of the smaller time window), it is more challenging for the solver to search for the optimal solution. Concretely, most of its computing time is used to prove the solution's optimality. This means that if a sub optimal solution, with an integrity gap, is acceptable, then the required solution time could be much less.

\section{B. Overall results comparison}

The main indices of the results are summarized in Table III. For Model DCB, we can see that using only ground holding to balance demand with capacity needs huge amount of delays. Moreover, due to some long delays, many demands are actually moved to the next day (i.e., 27,654 - 26,371) which we assume has unlimited capacities. If they are further imposed, along with the next day's traffic, the required delays could be even higher. Nevertheless, it is worth noting that in realities there will never be such an amount of delays because in many cases some capacity overloads will be allowed. and sometimes the allowance could be quite large. In this study, we consider a relatively conservative situation, with only $10 \%$ maximal capacity allowance, for the illustrative purpose.

For Model C-DCB, a promising finding is that delays are reduced significantly to 3,402 mins in total (see Table III), because of using the alternative trajectory options. But we can notice from the table that most of the flights still keep their initially scheduled trajectories $(5,741)$ which accounts for $92 \%$ of the total flights. In other words, only $8 \%$ of flights diverted to their (preferred) alternative trajectories could contribute to a reduction of $98 \%$ of total delays. This is because only assigning delays in a network scenario will normally cause inefficient usage $(57.7 \%)$ of some airspace capacities, while allowing alternatives could take advantage of the capacities in those less-congested areas.

However, the most notable finding is for Model SC-DCB, in which delays are further reduced, more flights can use initial trajectories, and, moreover, less sectors are opened and less amount of total capacity provisions are needed. This reveals the obvious effects of adjusting sector opening schemes synchronously along with the changes of traffic flow.
TABLE III

OVERALL RESULTS COMPARISON BETWEEN THE THREE MODELS.

\begin{tabular}{c|c|c|c}
\hline Index & Model DCB & Model C-DCB & Model SC-DCB \\
\hline Delays (min) & 185,263 & 3,402 & 381 \\
\hline Delayed flights & 1,353 & 417 & 167 \\
\hline Initial trajectory & 6,255 & 5,741 & 5,755 \\
\hline Lateral altern. & 0 & 265 & 246 \\
\hline Vertical altern. & 0 & 249 & 254 \\
\hline Total capacity & 45,708 & 45,708 & 33,545 \\
\hline Total \# sectors & 1,098 & 1,098 & 773 \\
\hline Pre demand & 27,654 & 27,654 & 27,654 \\
\hline Post demand & 26,371 & 27,242 & 24,840 \\
\hline Post D/C ratio & $57.7 \%$ & $59.6 \%$ & $74.0 \%$ \\
\hline
\end{tabular}

Specifically, as shown in Table III, a small number of delays (and delayed flights) exist in this model, being $381 \mathrm{~min}$ (and 167 flights) in total, which accounts for only around $11 \%$ of that assigned in model C-DCB. Besides, the number of flights using alternative trajectories also decreases by 14 flights.

Then, in terms of capacity, it is typically known that the more capacities we can make use of, the less delays there will be, but in this particularity case, the total capacity even reduces by $19 \%$. Meanwhile, the number of opened sectors reduces too by around 30\% from 1,098 to 773 .

On the other hand, as mentioned previously, the adjustment of sector collapsing affects traffic demand counting, such that the more elementary sectors are merged into one operating sector, the less traffic demand (i.e., flight entry) will be counted. This is also observed in Table III where the traffic demand reduces by $10 \%$ to 24,840 . Nevertheless, the average capacity usage increases to $74 \%$, much higher than the numbers for the previous two models, which turns to be the main reason that causes less traffic flow regulations in this model.

\section{Demand and capacity}

The demand and capacity situations are shown in Fig. 7 with respect to the original and those after executing the three models. We can see from Fig. 7(a) that, across the 1,098 operating sectors in total, a certain amount of capacity overloads occur for some sectors. Moreover, in some cases, the traffic demand could be more than double of the capacity. That is to say, for the purpose of balancing demand with capacity in Model DCB (see Fig. 7(b)), we have to delay half of the flights traversing this sector, and the delayed flights will often incur new delays in other (near) congested sectors. This accumulative effect may easily evolve to large amount of total delays which we have seen in Table III for Model DCB.

Allowing alternative trajectories in Model C-DCB is obviously one way to leverage the above delay accumulation, because diverting flights to some less-congested sectors does not necessarily generate new delays. As shown in Fig. 7(c), some free sectors with low traffic demand in Fig. 7(b) is now filled with relatively high traffic demand. This can be seen more clearly by the demand and capacity ratio shown in Fig. 8(a), namely the blue line versus the red line. It is worth noting that even with this slight improvement, the delays can be reduced remarkably (recall Table III). Nevertheless, we may 


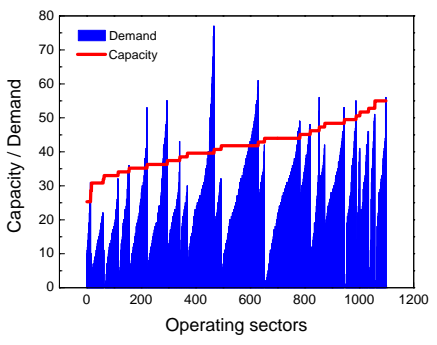

(a) Original situation

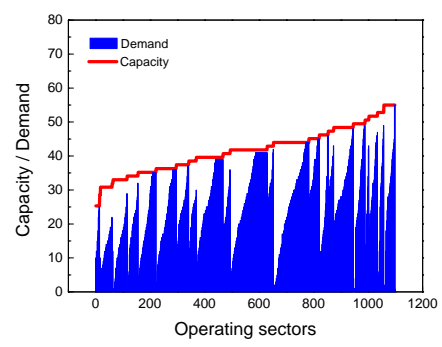

(c) Model C-DCB

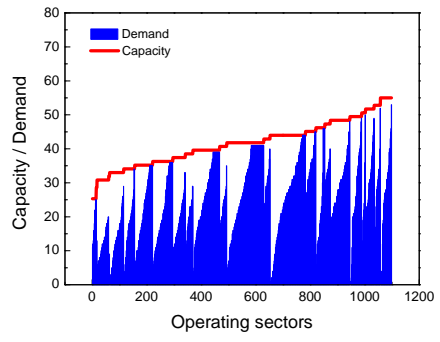

(b) Model DCB

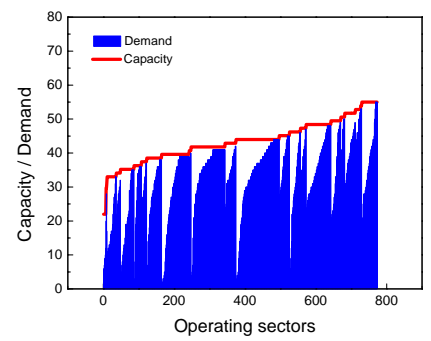

(d) Mode SC-DCB
Fig. 7. Demand and capacity situations in the original and three models.

still see may blank areas in Fig. 7(c) underneath the capacity line, meaning that many capacities are not well utilized.

The last Model SC-DCB solves this issue quite well, as proved by the results in Fig. 7(d). The number of opened sectors are reduced to 773 , and all the traffic demand is compacted to the lessen area, which in turn leaves less blanks left. Meanwhile, Fig. 8(a) presents a large improvement of the capacity load for Model SC-DCB (green line) if compared to the other two models.

Finally, we can notice that not only the average value increases, but also most of the sectors $(75 \%)$ have their capacity loads greater than $60 \%$. This number for Model DCB and C-DCB is only around $40 \%$. On the other side, there are almost 100 sectors in both Model DCB and C-DCB that have a capacity load less than $10 \%$ (with some $0 \%$ cases), which is quite low and not an expected situation even from the safety aspect. But in Model SC-DCB, we can see only quite few sectors having such low capacity loads.

\section{Sector opening scheme}

In the previous Sec. VI-C, we have demonstrated that Model SC-DCB enables a notable improvement on the utilization of capacity resources. This is realized by optimizing sector opening schemes and traffic flow (including trajectory selections and delay assignments) in a synchronized way.

Fig. 9(a) shows the changes of number of opened sectors during each time period of the day, and we can observe the number reduction for every time period in Model SC-DCB. In the objective function (15) of this model, we minimize the total number of opened sectors (as one of the multi objectives). This is because: 1) it is directly related to the ATC system costs, and 2) less number of sectors means larger size to each of them which means the traffic demand could be further compacted (recall Fig. 7(d)) to reduce the percentage of "idle" capacity.

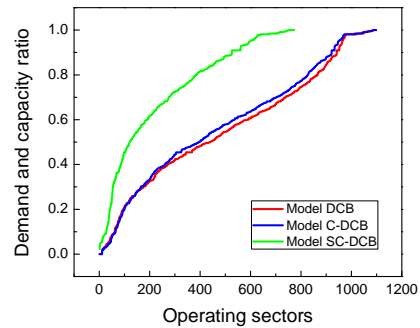

(a) Sorted capacity load

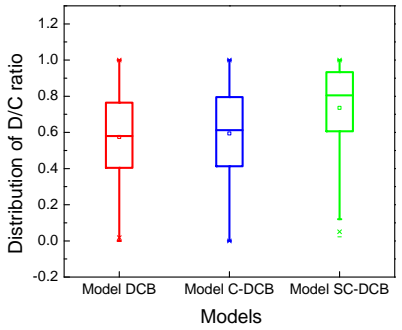

(b) Distribution of capacity load
Fig. 8. Final capacity load (i.e., demand and capacity ratio) in three models.

However, the number of opened sectors cannot be reduced unlimitedly, as more traffic in less sectors will soon cause the capacities to be fully taken or even overloaded.

Following the reduced amount of operating sectors, the total capacity provisions are lowered down as well, as shown in Fig. 9(b). Given most of the sectors' capacities are usually not varied too much, the changes of capacity provisions are basically in line with the number of opened sectors.

For the average capacity provision per opened sector (see Fig. 9(c)), the changes are worth noting. We can see that during the periods when there are less traffic (typically from 0-6 hour and 23-24 hour in a day), the original setting provides a higher average capacity, but during the congested periods, it somehow gives a relatively lower average value (see the gray line in Fig. 9(c)). This is because the original setting relies on cutting airspace into smaller pieces of sectors (each with lower capacity) to better manage the traffic flow.

On the contrary, Model SC-DCB provides an average capacity almost in consistent with the number of sectors and the

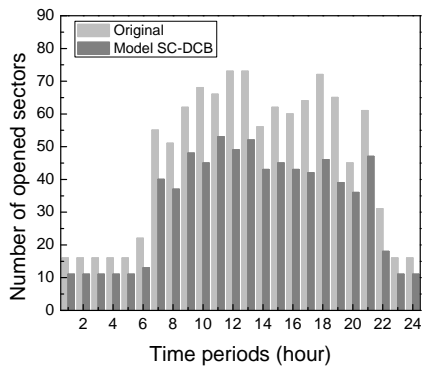

(a) Number of opened sectors

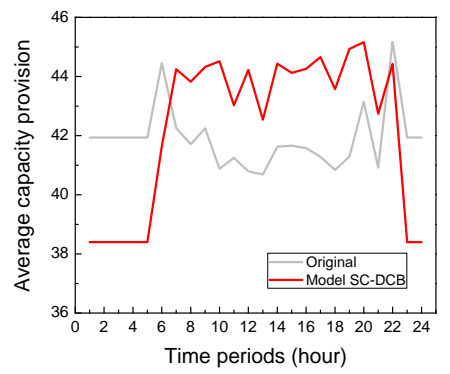

(c) Average capacity provision

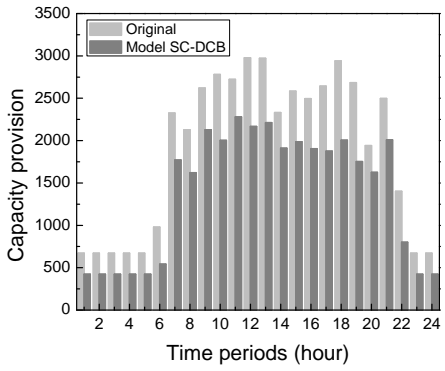

(b) Total capacity provision

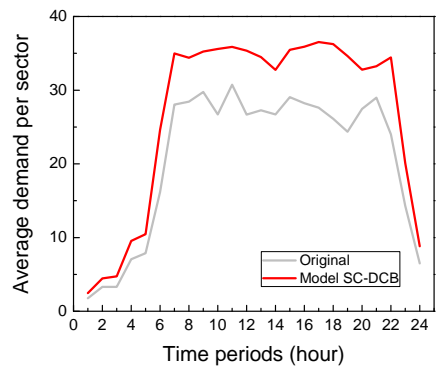

(d) Average demand per sector
Fig. 9. Changes of sector opening schemes in Model SC-DCB. 
TABLE IV

AirspaCe CONFIgURATIONS ACTIVATED IN MOdel SC-DCB FOR EACH ACC DURING EACH TIME PERIOD OF THE DAY

\begin{tabular}{|c|c|c|c|c|c|c|c|c|c|c|c|c|c|c|c|}
\hline Time & LFBBCTA & LFEECTAC & LFEECTAE & LFEECTAN & LFFFCTAA & LFFFCTAE & LFFFCTAW & LFMLTMA & LFMMCTAE & LFMMCTAW & LFMMXCTA & LFRRCTAE & LFRRCTAN & LFRRCTAS & LFSBTMA \\
\hline 1 & 1.A & ICA & $1 \mathrm{~EB}$ & $1 \mathrm{NB}$ & 1 & $1 \mathrm{~A}$ & $1 \mathrm{~A}$ & C1A & E1A & W1A & CF1 & C01EA & C01NA & C01SFS & ALL \\
\hline 2 & $1 . \mathrm{S}$ & $1 \mathrm{CC}$ & $1 \mathrm{~EB}$ & $1 \mathrm{NB}$ & 1 & $1 \mathrm{~A}$ & $1 \mathrm{~A}$ & $\mathrm{ClA}$ & E1A & W1A & CF1 & C01EFS & C01NFS & C01SA & ALL \\
\hline 3 & 1.A & $1 \mathrm{CA}$ & 1EA & INA & 1 & $1 \mathrm{~A}$ & $1 \mathrm{~A}$ & C1A & E1A & W1A & CF1 & C01EFS & CO1NA & C01SA & ALL \\
\hline 4 & $1 . \mathrm{S}$ & $1 \mathrm{CB}$ & 1EA & 1NA & 1 & $1 \mathrm{~A}$ & $1 \mathrm{~A}$ & $\mathrm{C} 1 \mathrm{~A}$ & E1A & W1A & $\mathrm{CF} 1$ & C01EA & CO1NA & Co1SA & ALL \\
\hline 5 & 1.A & $1 \mathrm{CB}$ & $1 \mathrm{EC}$ & 1NA & 1 & $1 \mathrm{~A}$ & $1 \mathrm{~A}$ & $\mathrm{C} 1 \mathrm{~A}$ & E1A & W1A & $\mathrm{CF} 1$ & C01EA & C01NA & C01SFS & ALL \\
\hline 6 & 3.1AN & $1 \mathrm{CC}$ & $1 \mathrm{EC}$ & 1NA & 1 & $1 \mathrm{~A}$ & $1 \mathrm{~A}$ & C1A & E1A & W1A & $\mathrm{CF} 1$ & C01EA & C01NFS & C01SA & ALL \\
\hline 7 & $9.1 \mathrm{~A}$ & $3 \mathrm{CA}$ & $2 \mathrm{~EB}$ & $4 \mathrm{NC}$ & 1 & $5 \mathrm{C}$ & $2 \mathrm{~A}$ & $\mathrm{C} 1 \mathrm{~A}$ & E4A & W2A2A & CF1 & CO5EZZ & C01NFS & C01SA & ALL \\
\hline 8 & $7.1 \mathrm{D}$ & $2 \mathrm{CA}$ & $2 \mathrm{~EB}$ & $5 \mathrm{NA}$ & 1 & $3 \mathrm{~A}$ & $4 \mathrm{C}$ & ClA & E2B & W2B $1 \mathrm{~A}$ & $\mathrm{CF} 1$ & C04EX & $\mathrm{C} 03 \mathrm{NC}$ & C02SA & ALL \\
\hline 9 & 14.1P & $2 \mathrm{CA}$ & $2 \mathrm{~EB}$ & $4 \mathrm{NC}$ & 1 & $5 \mathrm{C}$ & $3 \mathrm{~A}$ & $\mathrm{C} 1 \mathrm{~A}$ & E4A & W3B2A & CF1 & CO5EZZ & C02NA & C02SFS & ALL \\
\hline 10 & $9.1 \mathrm{D}$ & $3 \mathrm{CA}$ & $2 \mathrm{~EB}$ & 5NA & 1 & $4 \mathrm{C}$ & $4 \mathrm{~A}$ & C1A & $\mathrm{E} 4 \mathrm{~A}$ & W2A2A & CF1 & C05EY & $\mathrm{C} 03 \mathrm{NC}$ & C02SFS & ALL \\
\hline 11 & $12.1 \mathrm{G}$ & $3 \mathrm{CA}$ & $2 \mathrm{~EB}$ & $4 \mathrm{NC}$ & 1 & $5 \mathrm{C}$ & $3 \mathrm{~A}$ & $\mathrm{ClA}$ & E4B $1 A$ & W4A1A & $\mathrm{CF} 1$ & C07EK & C04NA & $\mathrm{C} 03 \mathrm{SC}$ & ALL \\
\hline 12 & $12.1 \mathrm{G}$ & $2 \mathrm{CA}$ & $2 \mathrm{~EB}$ & $4 \mathrm{NB}$ & 1 & $5 \mathrm{~F}$ & $3 \mathrm{~A}$ & $\mathrm{C} 1 \mathrm{~A}$ & E4B $1 \mathrm{~A}$ & W3B1A & CF1 & CO5EZZ & C04NA & C03SA & ALL \\
\hline 13 & $12.1 \mathrm{G}$ & $3 \mathrm{CA}$ & $2 \mathrm{~EB}$ & 7NA & 1 & $5 \mathrm{~F}$ & $4 \mathrm{~A}$ & $\mathrm{C} 1 \mathrm{~A}$ & E4A & W2A1A & $\mathrm{CF} 1$ & C07EG & $\mathrm{C} 03 \mathrm{NC}$ & C02SA & ALL \\
\hline 14 & $12.1 \mathrm{G}$ & $2 \mathrm{CA}$ & $2 \mathrm{~EB}$ & $5 \mathrm{NN}$ & 1 & $3 \mathrm{~A}$ & $2 \mathrm{~A}$ & $\mathrm{C} 1 \mathrm{~A}$ & E3C & W3A1A & CF1 & C04EX & $\mathrm{C} 04 \mathrm{NC}$ & C02SFS & ALL \\
\hline 15 & $10.1 \mathrm{E}$ & $3 \mathrm{CA}$ & $2 \mathrm{~EB}$ & 5NA & 1 & $5 \mathrm{~F}$ & $2 \mathrm{~A}$ & C1A & $\mathrm{E} 4 \mathrm{E}$ & W3B2A & CF1 & C04EA & C02NFS & C03SA & ALL \\
\hline 16 & $12.1 \mathrm{G}$ & $2 \mathrm{CA}$ & $2 \mathrm{~EB}$ & $5 \mathrm{ND}$ & 1 & $4 \mathrm{~A}$ & $3 \mathrm{~A}$ & $\mathrm{ClA}$ & E3C & W2AlA & CF1 & CO5EZZ & $\mathrm{C} 02 \mathrm{NA}$ & C02SA & ALL \\
\hline 17 & $12.1 \mathrm{G}$ & $2 \mathrm{CA}$ & $2 \mathrm{~EB}$ & $4 \mathrm{NC}$ & 1 & $3 \mathrm{~A}$ & $2 \mathrm{~A}$ & $\mathrm{C} 1 \mathrm{~A}$ & E4A & W3B1A & CF1 & C04EA & $\mathrm{C} 02 \mathrm{NA}$ & C03SA & ALL \\
\hline 18 & $14.1 \mathrm{~K}$ & $2 \mathrm{CA}$ & $2 \mathrm{~EB}$ & $4 \mathrm{NH}$ & 1 & $3 \mathrm{~A}$ & $2 \mathrm{~A}$ & C1A & E3C & W2A $2 \mathrm{~A}$ & CF1 & C07EE & C02NA & C03SC & ALL \\
\hline 19 & $10.1 \mathrm{E}$ & $2 \mathrm{CA}$ & $2 \mathrm{~EB}$ & $4 \mathrm{NH}$ & 1 & $4 \mathrm{C}$ & $3 \mathrm{~B}$ & $\mathrm{C} 1 \mathrm{~A}$ & $\mathrm{E} 3 \mathrm{C}$ & W $2 \mathrm{~A} 1 \mathrm{~A}$ & CF1 & C04EX & C02NA & C02SFS & ALL \\
\hline 20 & $12.1 \mathrm{G}$ & $2 \mathrm{CA}$ & $1 \mathrm{~EB}$ & $4 \mathrm{NB}$ & 1 & $3 \mathrm{~A}$ & $2 \mathrm{~A}$ & C1A & $\mathrm{E} 2 \mathrm{~B}$ & W2A $2 A$ & CF1 & $\mathrm{C} 03 \mathrm{EF}$ & C01NFS & C02SFS & ALL \\
\hline 21 & $12.1 \mathrm{G}$ & $3 \mathrm{CA}$ & $4 \mathrm{EK}$ & $5 \mathrm{NA}$ & 1 & $5 \mathrm{~F}$ & $4 \mathrm{~A}$ & $\mathrm{C} 1 \mathrm{~A}$ & E2B & W2A1A & $\mathrm{CF} 1$ & C05EX & $\mathrm{C} 02 \mathrm{NA}$ & $\mathrm{C} 02 \mathrm{SA}$ & ALL \\
\hline 22 & $5.1 \mathrm{D}$ & $1 \mathrm{CC}$ & $1 \mathrm{~EB}$ & $1 \mathrm{NB}$ & 1 & $1 \mathrm{~A}$ & $1 \mathrm{~A}$ & $\mathrm{C} 1 \mathrm{~A}$ & $\mathrm{E} 2 \mathrm{~B}$ & W2A1A & $\mathrm{CF} 1$ & CO1EA & CO1NA & C01SA & ALL \\
\hline 23 & 1.A & $1 \mathrm{CC}$ & IEA & $1 \mathrm{NB}$ & 1 & $1 \mathrm{~A}$ & $1 \mathrm{~A}$ & C1A & E1A & W1A & CF1 & C01EA & CO1NA & C01SFS & ALL \\
\hline 24 & $1 . \mathrm{S}$ & $1 \mathrm{CB}$ & $1 \mathrm{EC}$ & 1NA & 1 & $1 \mathrm{~A}$ & $1 \mathrm{~A}$ & C1A & E1A & W1A & CF1 & C01EFS & COINA & C01SFS & ALL \\
\hline
\end{tabular}

capacity provisions (see the red line), meaning that the opened sectors in this model share similar unit capacities. Moreover, the unit capacities are also higher than those of the smaller sectors used in the original setting, and thus more demand can be accommodated per sector, as shown in Fig. 9(d).

The final configuration for each ACC of the study is presented in Table IV. We can see the choice of configuration, for some ACCs, evolves during different time periods of the day. Nevertheless, as mentioned in V-B, we consider in this paper a limited dynamic sectorization which is subject to the existing airspace configurations. This means that both the collapsing architecture of elementary sectors and the opening schemes of operating sectors must follow certain groups of rules. In future work, a more flexible sectorization method with such constraints relaxed should be further taken into account.

\section{CONCLUSIONS}

This paper presented a preliminary method of synchronizing the planning of traffic flow and sector opening. Through combining different traffic management initiatives - such as assigning ground delays and diverting flights to alternative trajectories - along with the dynamic sectorization measures into an integrated optimization model, the performance of demand and capacity balancing can be improved remarkably. Results show that not only the system delays can be largely reduced, but also the ATC operational costs and the required total capacity provisions. Future work will focus on extending the method to further take uncertainties into account.

\section{ACKNOWLEDGMENT}

The work was partially funded by grants from the CSC No 201506830050 and by the SESAR Joint Undertaking under grant agreement No 699338, as part of the European Unions Horizon 2020 research and innovation programme: APACHE project (Assessment of Performance in current ATM operations and of new Concepts of operations for its Holistic Enhancement - http://apache-sesar.barcelonatech- upc.eu/en). The opinions expressed herein reflect the authors view only. Under no circumstances shall the SESAR Joint Undertaking be responsible for any use that may be made of the information contained herein. The authors would like to thank Mr. Ramon Dalmau for generating the trajectory data and relevant figures.

\section{REFERENCES}

[1] Central Office for Delay Analysis, "All-causes delay and cancellations to air transport in Europe," Tech. Rep. CODA Digest 2016, CDA-2017005, Network Manager, 2017.

[2] D. Bertsimas and S. S. Patterson, "The air traffic flow management problem with enroute capacities," Operations research, vol. 46 , no. 3 , pp. 406-422, 1998.

[3] A. R. Odoni, "The flow management problem in air traffic control," in Flow control of congested networks, pp. 269-288, Springer, 1987.

[4] M. Terrab and S. Paulose, "Dynamic strategic and tactical air traffic flow control," in IEEE International Conference on Systems, Man and Cybernetics, Chicago, USA, pp. 243-248, IEEE, 1992.

[5] G. Lulli and A. Odoni, "The european air traffic flow management problem," Transportation Science, vol. 41, no. 4, pp. 431-443, 2007.

[6] D. Bertsimas and S. Gupta, "Fairness and collaboration in network air traffic flow management: an optimization approach," Transportation Science, vol. 50, no. 1, pp. 57-76, 2015.

[7] FAA, "Traffic Flow Management in the National Airspace System," Tech. Rep. FAA-2009-AJN-251, Federal Aviation Administration, 2009.

[8] FAA, "Collaborative Trajectory Options Program (CTOP): Document Information.," Tech. Rep. AC 90-115, Federal Aviation Administration, 2014.

[9] P. Kopardekar, K. Bilimoria, and B. Sridhar, "Initial concepts for dynamic airspace configuration," in Proceedings of 7th AIAA Aviation Technology, Integration and Operations Conference (ATIO, AIAA.

[10] H. D. Sherali and J. M. Hill, "Configuration of airspace sectors for balancing air traffic controller workload," Annals of Operations Research, vol. 203, no. 1, pp. 3-31, 2013.

[11] A. Klein, M. D. Rodgers, and H. Kaing, "Dynamic fpas: A new method for dynamic airspace configuration," in Integrated Communications, Navigation and Surveillance Conference, 2008. ICNS 2008, pp. 1-11, IEEE, 2008

[12] $\mathrm{Y}$. $\mathrm{Xu}$ and $\mathrm{X}$. Prats, "Including linear holding in air traffic flow management for flexible delay handling," Journal of Air Transportation, vol. 25, pp. 123-137, 2017.

[13] Y. Xu, R. Dalmau, M. Melgosa, A. de Montlaur, and X. Prats, "Alternative trajectories for delay reduction in demand and capacity balancing," in Proceedings of the 8th International Conference for Research in Air Transportation (ICRAT), (Castelldefels, Spain), 2018.

[14] D. Bertsimas, V. F. Farias, and N. Trichakis, "The price of fairness," Operations research, vol. 59, no. 1, pp. 17-31, 2011.

[15] R. Dalmau, M. Melgosa, S. Vilardaga, and X. Prats, "A fast and flexible aircraft trajectory predictor and optimiser for atm research applications," in 8th International Conference for Research in Air Transportation (ICRAT), (Castelldefels, Spain), 2018. 